OPEN ACCESS

Edited by:

Dong-Yup Lee,

Sungkyunkwan University,

South Korea

Reviewed by:

Francesco Vitali,

National Research Council (CNR), Italy

Seo-Young Park,

Tufts University, United States

${ }^{*}$ Correspondence:

Babak Momen

momeni@bc.edu

Specialty section:

This article was submitted to

Systems Microbiology,

a section of the journal

Frontiers in Microbiology

Received: 01 October 2020

Accepted: 18 January 2021

Published: 10 February 2021

Citation:

Dedrick S, Akbari MJ, Dyckman SK, Zhao N, Liu Y-Y and Momeni B (2021) Impact of Temporal pH Fluctuations on the Coexistence of Nasal Bacteria in an in silico

Community

Front. Microbiol. 12:613109 doi: 10.3389/fmicb.2021.613109

\section{Impact of Temporal pH Fluctuations on the Coexistence of Nasal Bacteria in an in silico Community}

\author{
Sandra Dedrick ${ }^{1}$, M. Javad Akbari', Samantha K. Dyckman ${ }^{1}$, Nannan Zhao², \\ Yang-Yu Liu² and Babak Momeni ${ }^{1 *}$ \\ ${ }^{1}$ Department of Biology, Boston College, Chestnut Hill, MA, United States, ${ }^{2}$ Channing Division of Network Medicine, \\ Brigham and Women's Hospital, Boston, MA, United States
}

To manipulate nasal microbiota for respiratory health, we need to better understand how this microbial community is assembled and maintained. Previous work has demonstrated that the $\mathrm{pH}$ in the nasal passage experiences temporal fluctuations. Yet, the impact of such $\mathrm{pH}$ fluctuations on nasal microbiota is not fully understood. Here, we examine how temporal fluctuations in $\mathrm{pH}$ might affect the coexistence of nasal bacteria in in silico communities. We take advantage of the cultivability of nasal bacteria to experimentally assess their responses to $\mathrm{pH}$ and the presence of other species. Based on experimentally observed responses, we formulate a mathematical model to numerically investigate the impact of temporal $\mathrm{pH}$ fluctuations on species coexistence. We assemble in silico nasal communities using up to 20 strains that resemble the isolates that we have experimentally characterized. We then subject these in silico communities to $\mathrm{pH}$ fluctuations and assess how the community composition and coexistence is impacted. Using this model, we then simulate $\mathrm{pH}$ fluctuations - varying in amplitude or frequencyto identify conditions that best support species coexistence. We find that the composition of nasal communities is generally robust against $\mathrm{pH}$ fluctuations within the expected range of amplitudes and frequencies. Our results also show that cooperative communities and communities with lower niche overlap have significantly lower composition deviations when exposed to temporal $\mathrm{pH}$ fluctuations. Overall, our data suggest that nasal microbiota could be robust against environmental fluctuations.

Keywords: microbial communities, variable environment, nasal microbiota, mathematical model, species interaction network, community ecology, coexistence

\section{INTRODUCTION}

Resident microbes in the human nasal passage protect us from respiratory pathogens (Brugger et al., 2016; Man et al., 2017). Indeed, previous research shows the role of resident commensals in suppressing pathogens, such as Staphylococcus aureus (Uehara et al., 2000; Iwase et al., 2010; Bomar et al., 2016). Investigating how this microbial community is formed and maintained can therefore 
provide powerful insights into microbiota-based therapies to prevent or treat infections. While such an investigation appears formidable in complex environments such as the gut microbiota, it is feasible for nasal microbiota. First, the nasal microbiota has relatively low diversity, with the majority of composition often attributed to $3 \sim 8$ species (Escapa et al., 2018). Second, the majority of these species are readily culturable aerobically in vitro under controlled environments (Kaspar et al., 2016; Escapa et al., 2018). Third, both the species and the nasal environment can be sampled relatively easily (Yan et al., 2013; Proctor and Relman, 2017). The combination of these factors makes the nasal microbiota a suitable choice for mechanistic studies of human microbiota and a gateway for more detailed studies of humanassociated microbiota. Despite these advantages, communitylevel modeling of nasal microbiota has not been discussed adequately so far. A majority of existing work has focused on the biology of specific members of the nasal microbiota such as Staphylococcus aureus or Streptococcus pneumoniae because of their disease relevance (Regev-Yochay et al., 2004; Wertheim et al., 2004; Cespedes et al., 2005). Other reports have characterized and investigated the interactions among nasal microbes (Iwase et al., 2010; Bomar et al., 2016), but often with a focus on the interaction itself, and have only rarely involved the ecological consequences for the community (see Margolis et al., 2010; Yan et al., 2013; Krismer et al., 2017, for example).

Many factors, including interspecies interactions (Bomar et al., 2016; Brugger et al., 2016, 2020), the host immune system (Johannessen et al., 2012), and resource availability and access (Relman, 2012) can impact the nasal microbiota. However, all these factors take place in an environment that may fluctuate over time and vary in space. Previous investigations have revealed that the nasal passage is in fact very heterogeneous, both spatially and temporally (Proctor and Relman, 2017). In particular, $\mathrm{pH}$ fluctuations (in the range of 5.8-7.2, depending on the sampling site and time) were observed within the nasal passage (Washington et al., 2000; Hehar et al., 2001). Previous studies also demonstrate that temporal environmental fluctuations can transition the community to a different state (Abreu et al., 2020) or increase and support biodiversity (Eddison and Ollason, 1978; Grover, 1988; Abrams and Holt, 2002; Jiang and Morin, 2007; Kremer and Klausmeier, 2013). The explanation is often based on the temporal niche partitioning mechanism; i.e., environment variations creates additional niches and allow for more species to coexist (Chesson, 2000; Amarasekare, 2003). The purpose of our work is not to introduce a new theoretical framework for modeling microbial communities. Instead, we aim for a predictive mathematical model to study the impact of temporal $\mathrm{pH}$ fluctuations on the nasal microbiota composition. Other factors notwithstanding, we specifically ask whether, and when, incorporating temporal $\mathrm{pH}$ fluctuations is necessary to accurately predict compositional outcomes.

To answer the above question, we first characterize six nasal bacterial isolates as representative of members present in the nasal community. The rationale behind choosing these nasal bacteria was that (1) we can culture these strains reliably in the same cultivation medium and conditions in the lab; (2) covering different Corynebacterium and Staphylococcus species, these strains capture some of the natural diversity of microbiota (Escapa et al., 2018); and (3) Some interactions among these strains has already been identified (Brugger et al., 2020). For instance, Corynebacterium have been used to inhibit S. aureus colonization (Uehara et al., 2000; Kiryukhina et al., 2013) and $S$. aureus promotes the growth of $C$. accolens and gets inhibited by C. pseudodiphtheriticum (Yan et al., 2013). We then use in vitro communities constructed from nasal isolates to quantify the community response to temporal $\mathrm{pH}$ variations. Then, with parameters relevant to nasal microbiota, we use a phenomenological model to represent microbes and their interactions in an environment with a temporally fluctuating $\mathrm{pH}$. Based on our empirical characterizations of nasal bacteria, we construct in silico examples of nasal microbiota and quantify their response to temporal $\mathrm{pH}$ fluctuations. Our simulation results suggest that temporal $\mathrm{pH}$ fluctuations do not have a major impact on the stable coexistence of nasal bacteria. The outline of our procedure to assess the impact of temporal $\mathrm{pH}$ fluctuations on nasal microbiota is shown in Figure 1.

\section{MATERIALS AND METHODS}

\section{Nasal Bacterial Strains}

Six strains used in this study were isolated from two healthy individuals and kindly shared with us by Dr. Katherine Lemon (Table 1). Interactions between some of these strains and other nasal bacteria has been studied recently (Brugger et al., 2020).

\section{Cultivation Conditions and Medium in vitro}

As growth medium, we have used a 10-fold dilution of the ToddHewitt broth with yeast extract (THY, at an initial $\mathrm{pH}$ of 7.2). We have diluted THY to create an environment closer to the nutrient richness of the nasal passage (Krismer et al., 2014). For collecting cell-free filtrates, cells were grown in $15 \mathrm{ml}$ of media in sterile 50 $\mathrm{ml}$ Falcon tubes with loose caps exposed to the room atmosphere. For growth rate and carrying capacity characterizations, cells were grown in flat-bottom 96-well plates. All cultures were grown at $37^{\circ} \mathrm{C}$ with continuous shaking at $250 \mathrm{rpm}$.

\section{Characterizing the $\mathrm{pH}$ Response of Nasal Isolates in vitro}

To assess the response of nasal strains, we grew them in 10\% THY after adjusting the $\mathrm{pH}$ within the biologically relevant range of 5.1 and 7.5 at 0.3 intervals ( $\mathrm{pH}$ buffered with $10 \mathrm{~g} / \mathrm{l}$ of MOPS). For each strain, we measured the growth rate at low population sizes (before nutrients become limiting or byproducts become inhibitory) and the final carrying capacity. These values were measured by growing replicates of each strain (typically 6 replicates) in 96-well microtiter plates incubated inside a Synergy Mx plate reader. Growth rate and carrying capacity were 


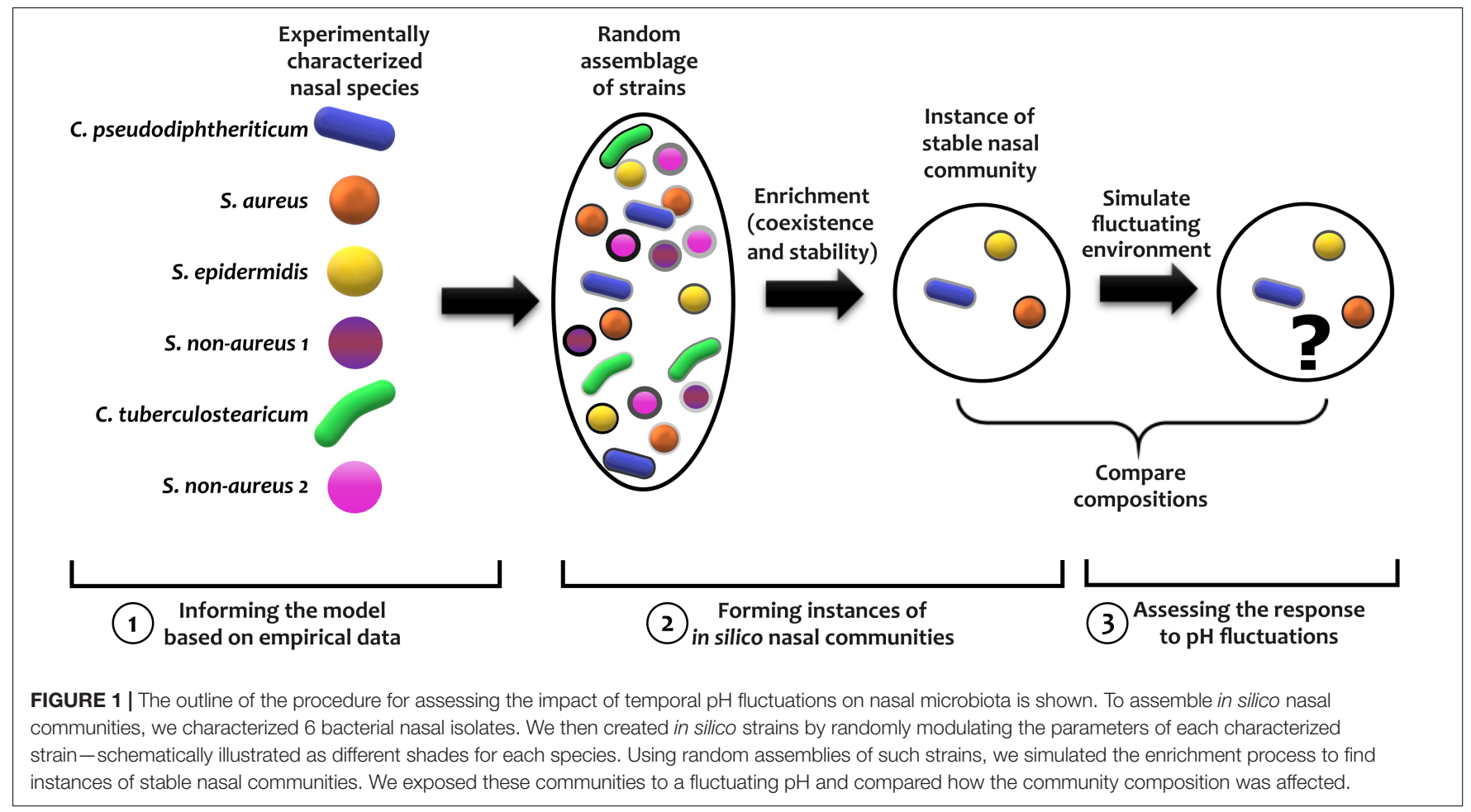

estimated by measuring the absorption in each well $\left(\mathrm{OD}_{600}\right)$ at 10 min intervals over $24 \mathrm{~h}$ at $37^{\circ} \mathrm{C}$. Between absorption reads, the plate was kept shaking to ensure a well-mixed environment.

\section{Mathematical Model}

To model the growth of species, we assume that in the absence of interactions, the population growth follows the logistic equations:

$$
\frac{d S_{i}}{d t}=r_{i}(p)\left[1-\frac{S_{i}}{K_{i}(p)}\right] S_{i}-\delta S_{i}
$$

In which $r_{i}(p)$ and $K_{i}(p)$ are the $\mathrm{pH}$-dependent growth rate and carrying capacity of species $i$. In our simulations, the growth rate and carrying capacity values at any given $\mathrm{pH}$ are found using a linear interpolation from experimentally measured values ( $\mathrm{pH} 5.1-7.5$ at 0.3 intervals). $\mathrm{pH}$ dependence is experimentally characterized for each strain in a monoculture, as described above, and $\delta$ is the dilution rate.

TABLE 1 | Nasal strains used in this study are listed along with their designation based on 16S rRNA gene similarity.

\begin{tabular}{lcc}
\hline Strain name & Genus & Most likely species designation \\
\hline KPL1821 & Corynebacterium & Corynebacterium tuberculostearicum \\
KPL1828 & Staphylococcus & Staphylococcus aureus \\
KPL1839 & Staphylococcus & Staphylococcus epidermidis \\
KPL1850 & Staphylococcus & Staphylococcus non-aureus 1 \\
KPL1989 & Corynebacterium & Corynebacterium pseudodiphtheriticum \\
KPL1867 & Staphylococcus & Staphylococcus non-aureus 2
\end{tabular}

When multiple species are present, we assume that the presence of other species takes away resources from the environment; as a result, the growth of each species will be modulated as

$$
\frac{d S_{i}}{d t}=r_{i}(p)\left[1-\frac{S_{i}-\gamma_{i}}{K_{i}(p)}\right] S_{i}-\delta S_{i} .
$$

where $\gamma_{i}=\sum_{j \neq i} c_{i j} S_{j}$ and $r_{i}(p) c_{i j} / K_{i}(p)$ represents the interaction strength exerted on species i by species j. Positive values of $c_{i j}$ indicate growth stimulation (e.g., via facilitation by producing resources) whereas negative values of $c_{i j}$ indicate growth inhibition (e.g., via competition).

\section{Model Parameters}

Unless otherwise specified, the following parameters are used in the model:

Some of these parameters, such as the range, frequency, and amplitude of $\mathrm{pH}$ values are chosen to keep the simulations close to what is expected in the nasal environment (Washington et al., 2000; Hehar et al., 2001). Some of the other parameters, such as the dilution rate or the initial and extinction population densities are not expected to be critical for the overall conclusions of this work. We have chosen these parameters to reflect realistic parameters that can be later tested experimentally. Finally, parameters such as the number of instances simulated $\left(N_{\mathrm{s}}\right)$ and the number of generations simulated $\left(N_{\text {gen }}\right)$ are chosen to give us enough confidence for our claims, while keeping the practical considerations of simulation time and effort in mind. 


\begin{tabular}{|c|c|c|}
\hline Parameter & Description & Value \\
\hline$N_{C}$ & $\begin{array}{l}\text { Maximum number of strains for in silico } \\
\text { community assembly }\end{array}$ & 20 \\
\hline$N_{S}$ & $\begin{array}{l}\text { Number of instances of assembly simulations } \\
\text { run for each case }\end{array}$ & 10,000 \\
\hline Ngen & $\begin{array}{l}\text { Number of generations simulated to obtain } \\
\text { stable resident communities; also the number } \\
\text { of generations simulated to assess response to } \\
\text { environmental fluctuations }\end{array}$ & 100 \\
\hline pHrng & $\begin{array}{l}\text { Range of } \mathrm{pH} \text { values (both in experiments and in } \\
\text { simulations) }\end{array}$ & $5.1-7.5$ \\
\hline$\delta$ & Dilution rate & $0.03-0.3 h^{-1}$ \\
\hline$N_{\text {ext }}$ & Extinction population density per species (OD) & $10^{-6}$ \\
\hline$f_{\mathrm{p}}$ & $\begin{array}{l}\text { Inter-strain parameter variation within each } \\
\text { species }\end{array}$ & $20 \%$ \\
\hline$s_{0}$ & Average initial cell density per strain (OD) & $10^{-4}$ \\
\hline$f_{\mathrm{pH}}$ & $\begin{array}{l}\text { Frequency of sinusoidal temporal pH } \\
\text { fluctuations }\end{array}$ & $1 h^{-1}$ \\
\hline$\Delta \mathrm{pH}$ & $\begin{array}{l}\text { Amplitude of sinusoidal temporal } \mathrm{pH} \\
\text { fluctuations }\end{array}$ & 0.5 \\
\hline
\end{tabular}

\section{Characterizing the Interspecies Interactions Using a Supernatant Assay}

To characterize how species $j$ affects the growth of other species $i$, we use a supernatant assay in which species $j$ is grown to saturation, then all the cells are filtered out using a $0.22 \mu \mathrm{m}$ filter (PVDF syringe filters from Thomas Scientific). The growth rate and carrying capacity of species $i$ is then measured when growing in the supernatant taken from cultures of species $j$. This formulation allows us to use the experimentally measurable supernatant responses to formulate a dynamical model for mixed cultures of multiple species.

Assuming a Lotka-Volterra model, the presence of another species modulates the growth rate proportionally to the size of the interacting partner, i.e.,

$$
\frac{d S_{i}}{d t}=r_{i}\left[1-\frac{S_{i}-c_{i j} S_{j}}{K_{i}}\right]\left(1-\frac{S_{i}-c_{i j} S_{j}}{K_{i}}\right) S_{i} .
$$

Calculating the parameters obtained from the cell-free spent media (CFSM), the carrying capacity for species $i$ is reached at population $S_{i, c c}$ level when growth rate becomes zero, thus

$$
\left(1-\frac{S_{i, c c}-c_{i j} K_{j}}{K_{i}}\right)=0 .
$$

Therefore, the carrying capacity in the supernatant assay $\left(K_{i j}\right)$ is

$$
K_{i j}=S_{i, c c}=K_{i}+c_{i j} K_{j} .
$$

And the interaction coefficient ( $c_{i j}$, effect of species $j$ on species $i$ ) can be calculated as

$$
c_{i j}=\frac{K_{i j}-K_{i}}{K_{j}} .
$$

In the particular that species $i$ and $j$ are similar (self-effect), we have $K_{i i}=0$ and $c_{i i}=-1$. It should be noted that there are limitations in using a Lotka-Volterra model. Such models may not accurately represent microbial interactions (Momeni et al., 2017). Additionally, under certain conditions, the solutions will exhibit instability. Particularly for this latter case, we examine the situations under which "runaway" growth instability may happen. Consider two mutualistic populations:

$$
\frac{d S_{1}}{d t}=r_{1}\left[1-\frac{S_{1}-c_{12} S_{2}}{K_{1}}\right] S_{1} \text { and } \frac{d S_{2}}{d t}=r_{2}\left[1-\frac{S_{2}-c_{21} S_{1}}{K_{2}}\right] S_{2}
$$

Instability can happen when the carrying capacity terms fail to act as a negative feedback to bound the population. This can happen when

$$
\left[1-\frac{S_{1}-c_{12} S_{2}}{K_{1}}\right]>1 \text { and }\left[1-\frac{S_{2}-c_{21} S_{1}}{K_{2}}\right]>1
$$

This happens when $S_{1}<c_{12} S_{2}$ and $S_{2}<c_{21} S_{1}$. Satisfying both of these inequalities requires that $c_{12}$ and $c_{12} c_{21}>1$, which means strong mutual facilitation. In our dataset, we do not have examples of mutual or cyclic facilitation and facilitation interaction terms are small, suggesting that instability is not expected in our simulations. Nevertheless, these conditions should be kept in mind for other datasets, especially those with strong facilitation between community members.

\section{Calculating Community Composition Deviations}

To compare community composition of a community that experienced $\mathrm{pH}$ fluctuation with that of the same community simulated at a fixed $\mathrm{pH}$, we calculated the Bray-Curtis dissimilarity measure using the f_dis function (option "BC") in MATLAB. The necessary files to reproduce the analysis are included in the accompanied source codes ${ }^{1}$.

\section{Estimating the Impact of pH Fluctuations}

We consider two extremes, when the fluctuations in $\mathrm{pH}$ are (1) much faster or (2) much slower than the population dynamics of community members. In both cases, for our formulation we define $c_{i i}=-1$ and use the simplified model of populations at different $\mathrm{pH}$ :

$$
\frac{d S_{i}}{d t}=r_{i}(p)\left[1+\frac{\widehat{\gamma_{i}}}{K_{i}(p)}\right] S_{i}-\delta S_{i}
$$

and

$$
\widehat{\gamma_{i}}=\sum c_{i j} S_{j}
$$

Case 1. Fast pH fluctuations: To estimate how the community responds under a rapidly changing $\mathrm{pH}$, we use the framework of the Wentzel-Kramers-Brillouin (WKB) approximation. For the general case of $\frac{d S}{d t}=r(t) S$, we split the population dynamics into two terms, the primary exponential term and an envelope

\footnotetext{
${ }^{1}$ https://github.com/bmomeni/temporal-fluctuations
} 
function, $E$, for which $E(t)=e^{-r_{0} t} S(t)$ and thus,

$$
\frac{d E}{d t}=\left[r(t)-r_{0}\right] E
$$

Using the WKB approximation $E$ can be written using the expansion

$$
E=\exp \left[\frac{1}{\varepsilon} \sum_{n=0}^{\infty} \varepsilon^{n} E_{n}(t)\right]
$$

By inserting this expansion into the differential equation, we obtain

$$
\begin{aligned}
& \left(\frac{1}{\varepsilon} \sum_{n=0}^{\infty} \varepsilon^{n} \frac{d}{d t} E_{n}(t)\right) \exp \left[\frac{1}{\varepsilon} \sum_{n=0}^{\infty} \varepsilon^{n} E_{n}(t)\right]= \\
& \left(r(t)-r_{0}\right) \exp \left[\frac{1}{\varepsilon} \sum_{n=0}^{\infty} \varepsilon^{n} E_{n}(t)\right]
\end{aligned}
$$

Thus

$$
\frac{1}{\varepsilon} \sum_{n=0}^{\infty} \varepsilon^{n} \frac{d}{d t} E_{n}(t)=r(t)-r_{0}
$$

Assuming sinusoidal changes in $\mathrm{pH}, p(t)=p_{0}+p_{d} \sin (2 \pi f t)$, to the first order, the temporal changes in growth rate can be approximated as, $r(t)=r_{0}+r_{d} \sin (2 \pi f t)$. Therefore,

$$
\frac{1}{\varepsilon} \sum_{n=0}^{\infty} \varepsilon^{n} \frac{d}{d t} E_{n}(t) \approx r_{d} \sin (2 \pi f t) .
$$

In the limit that $\varepsilon \rightarrow 0$ the first terms of expansion for $E$ are obtained as

$$
\begin{aligned}
& \frac{d}{d t} E_{0}(t)=0, \\
& \frac{d}{d t} E_{1}(t) \approx r_{d} \sin (2 \pi f t)
\end{aligned}
$$

Since the continuous dilutions in our setup keeps the populations finite, $E_{0}$ does not affect the solution. The dominant term for $E$ thus becomes $E_{1}$ and we have

$$
E_{1}(t) \approx \frac{-r_{d}}{2 \pi f} \cos (2 \pi f t)
$$

Aa a result,

$$
E(t) \propto \exp \left[\frac{-r_{d}}{2 \pi f} \cos (2 \pi f t)\right]
$$

Importantly, the magnitude of change in this equation drops inversely proportional to the frequency of $\mathrm{pH}$ fluctuations $f$. This means that the impact of $\mathrm{pH}$ fluctuations diminishes at high frequencies, consistent with our intuition that in this case the community dynamics are incapable of following the environmental fluctuations and only respond to the mean value.

Case 2. Slow pH fluctuations: In this case, we assume the quasistatic approximation, in which fluctuations are so slow that the community approaches its steady-state at each temporal value of $\mathrm{pH}$. In this situation, assuming $\frac{d S_{i}}{d t}=0$, we can rearrange the equation at steady-state as

$$
\left[r_{i}(p)-\delta\right] S_{i}=-\frac{r_{i}(p)}{K_{i}(p)} S_{i} \sum c_{i j} S_{j}
$$

Rearranging this, we get

$$
\left[\delta-r_{i}(p)\right] \frac{K_{i}(p)}{r_{i}(p)}=\sum c_{i j} S_{j} .
$$

This can be written in matrix form as

$$
[C] \underline{S}=\underline{b},
$$

where $[C]$ contains the interaction coefficients and $b_{i}=$ $\left[\delta-r_{i}(p)\right] \frac{K_{i}(p)}{r_{i}(p)}$; underline in our notation designates a vector. Since the interaction matrix $[C]$ is $\mathrm{pH}$-independent in our model, the change in composition within this quasi-static approximation can be expressed as

$$
[C]\left(\underline{S(t)}-\underline{S_{0}}\right)=\underline{b(t)}-\underline{b_{0}},
$$

or.

$$
\underline{\Delta S(t)}=\left(\underline{S(t)}-\underline{S_{0}}\right)=[C]^{-1}\left(\underline{b(t)}-\underline{b_{0}}\right)=[C]^{-1} \underline{\Delta b(t)}
$$

We make an additional simplifying assumption that $K_{i}$ and $r_{i}$ change similarly with $\mathrm{pH}$. This leads to $\Delta b_{i} \approx$ $\left(r_{i 0}-r_{i}(t)\right) \frac{K_{i 0}}{r_{i 0}}$. This means that the magnitude of change in community composition is the same as the change in the growth rate of species, regardless of the frequency of fluctuations, under this regime.

\section{Allowing pH-Dependent Interaction Coefficients}

To examine how $\mathrm{pH}$-dependent interaction coefficients may affect our results, we assumed that each interaction coefficient has a linear dependence on $\mathrm{pH}$ with a slope (per unit $\mathrm{pH}$ ) randomly selected from a uniform distribution in the range of $[-m, m]$. In other words,

$$
c_{i j}(p)=c_{i j}\left(p_{0}\right)+m\left(p-p_{0}\right)
$$

where $p_{0}=7.2$ is the $\mathrm{pH}$ at which our characterization is performed. We examined how the community composition deviated from the reference with a fixed $\mathrm{pH}$, as $m$ (and thus the $\mathrm{pH}$-dependency) increased.

\section{RESULTS}

\section{In vitro Characterization of Nasal Bacteria}

We experimentally characterized how six representative nasal bacterial strains respond to different $\mathrm{pH}$ values in their environment. These bacterial strains were chosen from a set of isolates (see section "Materials and Methods") based on three major considerations: (1) they reliably grow 
in our cultivation media under an aerobic environment; (2) they include commonly observed Staphylococcus and Corynebacterium species; and (3) they span the phylogenetic landscape of both closely and distantly related bacteria found in the nasal environment (Escapa et al., 2018). We assumed that each of these characterized strains is a representative strain of the corresponding species.

We first characterized the $\mathrm{pH}$ response of each strain by growing them under different environmental $\mathrm{pH}$ values. Different strains exhibited different degrees of $\mathrm{pH}$ dependency in their growth rates and carrying capacities (Figure 2 and Supplementary Figures S1, S2). Among these strains, S. epidermidis, $S$. non-aureus 1 , and $S$. non-aureus 2 show fairly similar growth properties. We chose to treat these as separate species in our investigation, because-as shown later-they had considerably different interactions with other species (Figure 3).

We then examined how different species interact with one another. For this, we grew each species to its stationary phase in a monoculture, filtered out the cells, and measured how other strains grew in the resulting cell-free filtrates (see section "Materials and Methods"; similar to De Vos et al., 2017; Brugger et al., 2020). From these measurements, we estimated the interspecies interaction coefficients based on the generalized LotkaVolterra model (Figure 3; see section "Materials and Methods"). In this formulation, baseline competition with complete niche overlap will result in an interaction coefficient of -1 . Of note, from our experimental data we cannot distinguish the relative contribution of competitive niche overlap and interspecies facilitation. Nevertheless, for simplicity we only use "facilitation" for extreme cases in which facilitation outweighs competition and the interaction coefficient turns positive. We interpret different gradations of negative interaction coefficients from -1 to 0 as different degrees of niche overlap (with -1 indicating complete niche overlap), and cases with interaction coefficients less than -1 indicate inhibition beyond competition for resources. Among the 30 pairwise interaction coefficients, there were 3 positive values (bright blue, marked by “ + " in Figure 3 ). For simplicity, throughout this manuscript, we assume that these interaction coefficients are not $\mathrm{pH}$-dependent.

\section{In silico Assembly of Nasal Bacterial Communities}

To capture some of the diversity of nasal microbiota, we propose that other in silico strains of each species can be constructed by randomly modulating the measured properties of that species (i.e., growth rate, carrying capacity, and interaction coefficients). We chose the degree of strain-level modulation to be up to $20 \%$, as a balance between intraspecies and interspecies diversity (Supplementary Figure S3).

To assess the response of nasal microbiota to temporal fluctuations in the environment, we first construct an ensemble of in silico communities that represent a subset of possible nasal communities. This is chosen as an alternative to performing an in vivo study, because performing these experiments with human subjects is not feasible and there is no reliable animal model for human nasal bacteria. Our approach is, in essence, similar to several other previous work that have used simple models to describe the dynamics of human-associated microbiota (Stein et al., 2013; Fisher and Mehta, 2014; Song et al., 2014; De Vos et al., 2017; Venturelli et al., 2018). Compared to in vitro studies, these in silico communities give us full control over confounding factors and allows us to examine the mechanisms contributing to sensitivity to $\mathrm{pH}$ fluctuations (Momeni et al., 2011). To construct in silico communities, we mimicked enrichment experiments (Goldford et al., 2018; Niehaus et al., 2019) by simulating the dynamics of an initial assemblage of 20 strains (sampled from the space of in silico strains) until the community reached stable coexistence. These in silico communities were largely robust against experimental noise in characterization (Supplementary Figure S4). The interspecies interactions in our model appear to be instrumental in the assembly of these in silico communities, as evidenced by changes when we assigned the interaction coefficients at given levels (Supplementary Figure S5A) or modulated the measured interactions (Supplementary Figure S5B). To assess how $\mathrm{pH}$ fluctuations in the environment influence nasal communities, we take several instances of in silico nasal communities, expose them to a fluctuating $\mathrm{pH}$, and quantify how the community composition is affected. The entire process is outlined in Figure 1.
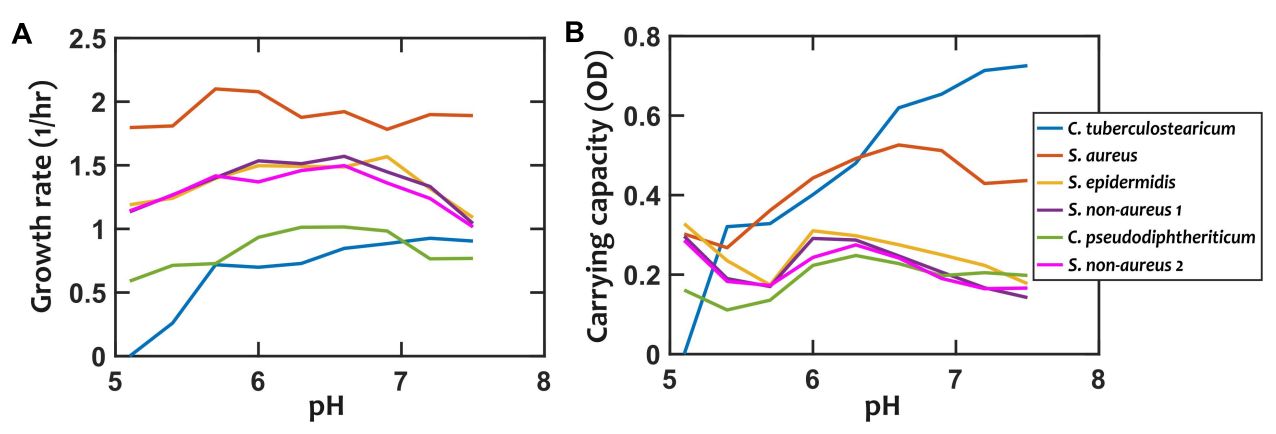

FIGURE 2 | Growth properties of nasal bacterial isolates are pH-dependent. Growth is characterized using the growth rate in the early exponential phase (A), and the carrying capacity based on optical density (OD, absorption measured at $600 \mathrm{~nm}$ ) as a proxy (B). Each data point is the average of at least 6 replicates from two independent experiments. Error-bars are not shown to avoid overcrowding the plot but the values are available in the raw data. In all cases, growth is experimentally tested in a 10-fold diluted Todd-Hewitt broth with yeast extract (10\% THY). 


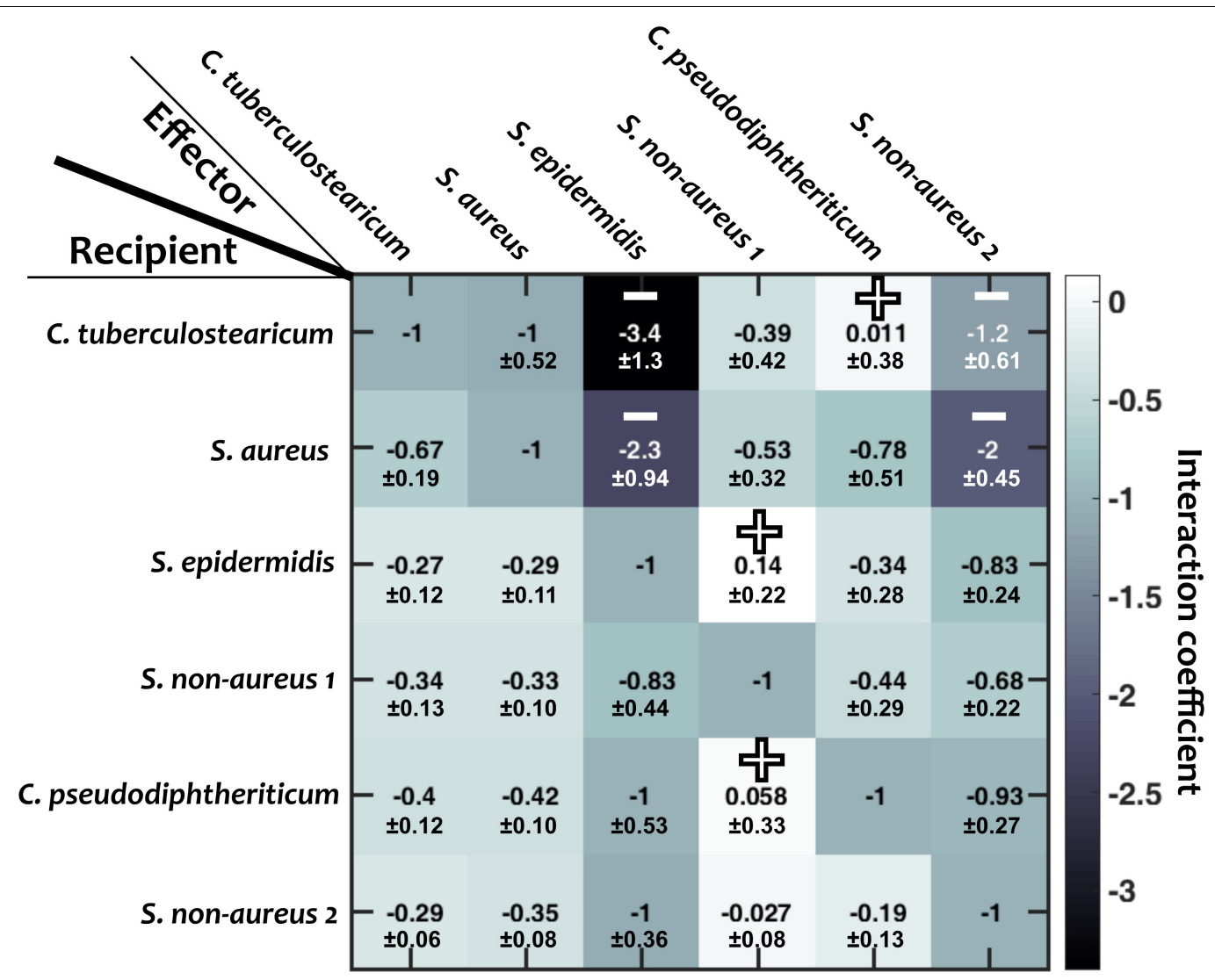

FIGURE 3 | Interaction coefficients among pairs of nasal bacteria. Values represent interaction coefficients in a Lotka-Volterra model. In each case, the growth of a recipient strain is measured when the strain is exposed to cell-free filtrate derived from the effector strain. Positive mean coefficients (indicating facilitation) and negative mean coefficients below -1.2 (indicating strong inhibition) across different replicates are marked by "+" and "-," respectively. Standard deviations (shown for each value) are calculated based on empirical standard deviations of measured carrying capacities in monocultures and supernatant experiments. Diagonal elements are set to -1 , indicating complete niche overlap.

\section{In silico Nasal Communities Are Diverse and Favor Facilitation}

We first examined the properties of assembled in silico communities at various $\mathrm{pH}$ values with no temporal fluctuations. We found that the prevalence of different species was distinct and $\mathrm{pH}$-dependent (Supplementary Figure S6). This prevalence is a result of nasal species' $\mathrm{pH}$-dependent growth properties as well as their interspecies interactions.

We also found that during the process of assembling in silico communities, the prevalence of interspecies facilitation interactions increased. Comparing the prevalence of facilitation in initial assemblages of strains vs. the final stable communities, we found that among the communities that had at least one facilitation interaction at the start of the in silico enrichment $(89 \%$ of communities), facilitation was enriched in $\sim 66 \%$ of the final community assemblies (Supplementary Figure S7).

\section{Temporal pH Fluctuations Only Minimally Impact Nasal Microbiota Composition}

Next, we asked how the temporal variation in the environment might influence the community composition. To answer this question, we used instances of in silico communities to evaluate the impact of temporal $\mathrm{pH}$ fluctuations. We assumed a continuous growth situation in which all community members experience a constant dilution rate. This dilution mimics the turnover in microbiota, for example, when the mucosal layer gets washed away. To avoid situations in which the in silico community itself was not stable, we changed the dilution rate by $\pm 50 \%$ and only kept the communities for which the modified dilutions only caused a small deviation in community composition (see section "Materials and Methods"). Indeed, we found that communities with compositions more sensitive to dilution rates are also more sensitive to $\mathrm{pH}$ fluctuations (Supplementary Figure S8). In all cases, composition deviations were calculated using the Bray-Curtis dissimilarity measure (see section "Materials and Methods").

To evaluate the impact of $\mathrm{pH}$ fluctuations, we simulated a controlled sinusoidal $\mathrm{pH}$ variation over time, with two parameters: the amplitude and frequency of temporal variations. Thus, $p(t)=p_{0}+\Delta \mathrm{pH} \sin \left(2 \pi f_{\mathrm{pH}} t\right)$. Keeping the frequency of fluctuations fixed $\left(f_{\mathrm{pH}}=0.2 / \mathrm{h}\right)$, we observed that the deviation in population composition increased with an increasing $\mathrm{pH}$ fluctuation amplitude $(\Delta \mathrm{pH})$. However, the resulting 

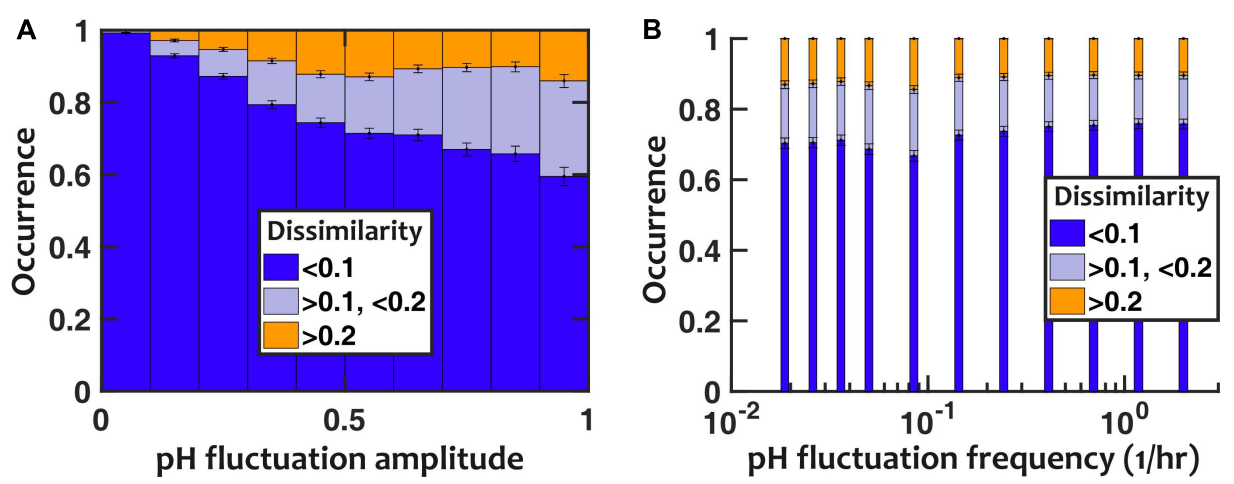

FIGURE 4 | Nasal microbiota composition is robust against pH fluctuations. (A) For a fixed fluctuation frequency $\left(f_{\mathrm{pH}}=0.2 / \mathrm{h}\right)$, larger fluctuation amplitudes increase how the community composition deviates from the no-fluctuation steady state (as quantified with composition dissimilarity). (B) The impact of temporal fluctuations is maximum at intermediate frequencies. Here, the $\mathrm{pH}$ fluctuation amplitude is fixed $(\Delta \mathrm{pH}=0.5)$. Number of in silico communities examined for each condition: $n=10,000$

dissimilarity in population composition was mostly minor, with $>85 \%$ of cases showing less than 0.2 dissimilarity even when the amplitude of $\mathrm{pH}$ fluctuation was set to 1 (Figure 4A). We then examined the impact of the frequency of $\mathrm{pH}$ variations, while we kept the amplitude of $\mathrm{pH}$ fluctuations fixed $(\Delta \mathrm{pH}=0.5)$. At intermediate frequencies, the $\mathrm{pH}$ fluctuations caused the largest dissimilarity in community composition compared to stable communities with fixed $\mathrm{pH}$ (Figure 4B).

We repeated the assessment of $\mathrm{pH}$ fluctuations by assuming a $\mathrm{pH}$ that randomly fluctuated between two discrete $\mathrm{pH}$ values to ensure that our results were not limited to sinusoidal fluctuations. The results were overall consistent with sinusoidal $\mathrm{pH}$ fluctuations (Supplementary Figure S9): (1) larger $\mathrm{pH}$ fluctuation amplitudes increased the deviation in population composition, but overall the majority of communities only experienced modest deviations; and (2) $\mathrm{pH}$ fluctuations at intermediate frequencies had the largest impact on community composition.

\section{Interspecies Facilitations Dampen the Impact of Temporal Fluctuations}

To explain the low sensitivity of community composition to $\mathrm{pH}$ fluctuations, we hypothesized that interspecies facilitation stabilizes the composition by creating interdependencies within the community. From the data in Figure 4, we picked and compared communities with low ("competitive" $0 \%$ facilitation) and high ("cooperative," $50 \%$ facilitation) prevalence of facilitation. The $0 \%$ facilitation corresponds to situations where none of the strains facilitate any of the other members of the in silico community. In contrast, $50 \%$ facilitation happens when half of the pairwise interactions among the in silico community are facilitative. Since in our dataset (Figure 3) there were no instances of mutual facilitation, $50 \%$ facilitation is the maximum fraction that cooperative communities can reach. The results show that cooperative communities have a consistently and significantly lower composition deviation when exposed to temporal pH fluctuations (Figure 5).

To further explore the impact of facilitation, we asked how interspecies niche overlap (the magnitude of negative interspecies interactions) and prevalence of facilitation (the fraction of interspecies interactions that are positive) contribute to sensitivity to $\mathrm{pH}$ fluctuations. In our results, we found that larger interspecies niche overlap leads to more sensitivity to $\mathrm{pH}$ fluctuations (Figure 6A). This trend holds except when interspecies niche overlap approaches 1 ; at such high overlaps the community loses diversity (Figure 6B), becoming less sensitive to $\mathrm{pH}$ fluctuations. When we directly changed the prevalence of facilitation, we observed that with higher prevalence of facilitation the communities became more diverse and less sensitive to $\mathrm{pH}$ fluctuations (Figures 6C,D).

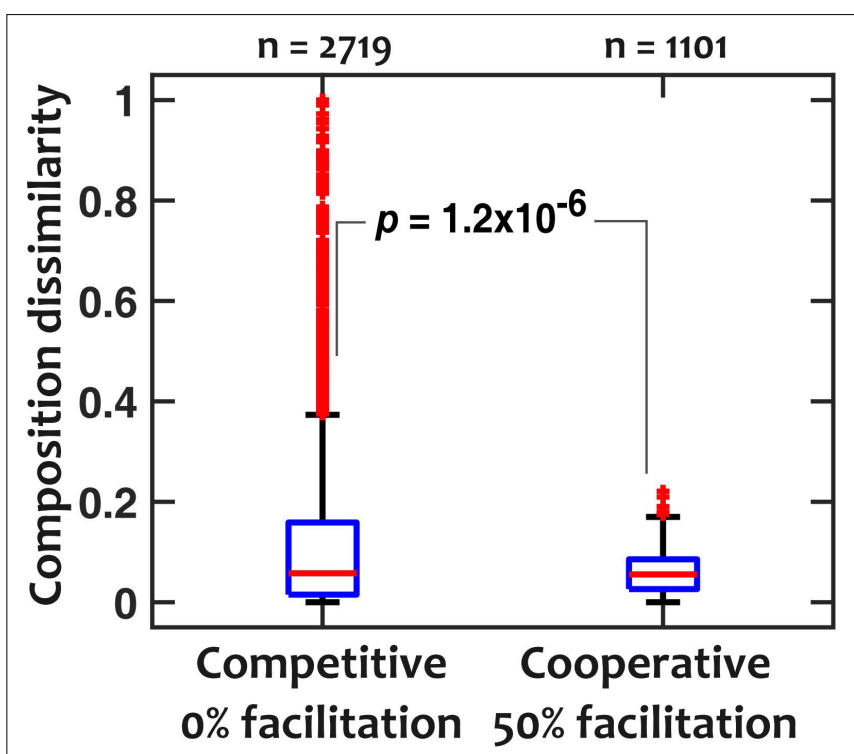

FIGURE 5 | Cooperative communities are more robust against $\mathrm{pH}$ fluctuations compared to competitive communities. For competitive (those with $0 \%$ facilitation among members) and cooperative (those with 50\% facilitation interactions among members) communities, dissimilarity medians were 0.057 and 0.055 and dissimilarity means were 0.12 and 0.058 , respectively $\left(p=1.2 \times 10^{-6}\right.$ with a Mann-Whitney $U$-test). $\mathrm{pH}$ fluctuates sinusoidally with a frequency of $f_{\mathrm{pH}}=0.2 / \mathrm{h}$ and an amplitude of $\Delta \mathrm{pH}=0.5$. 

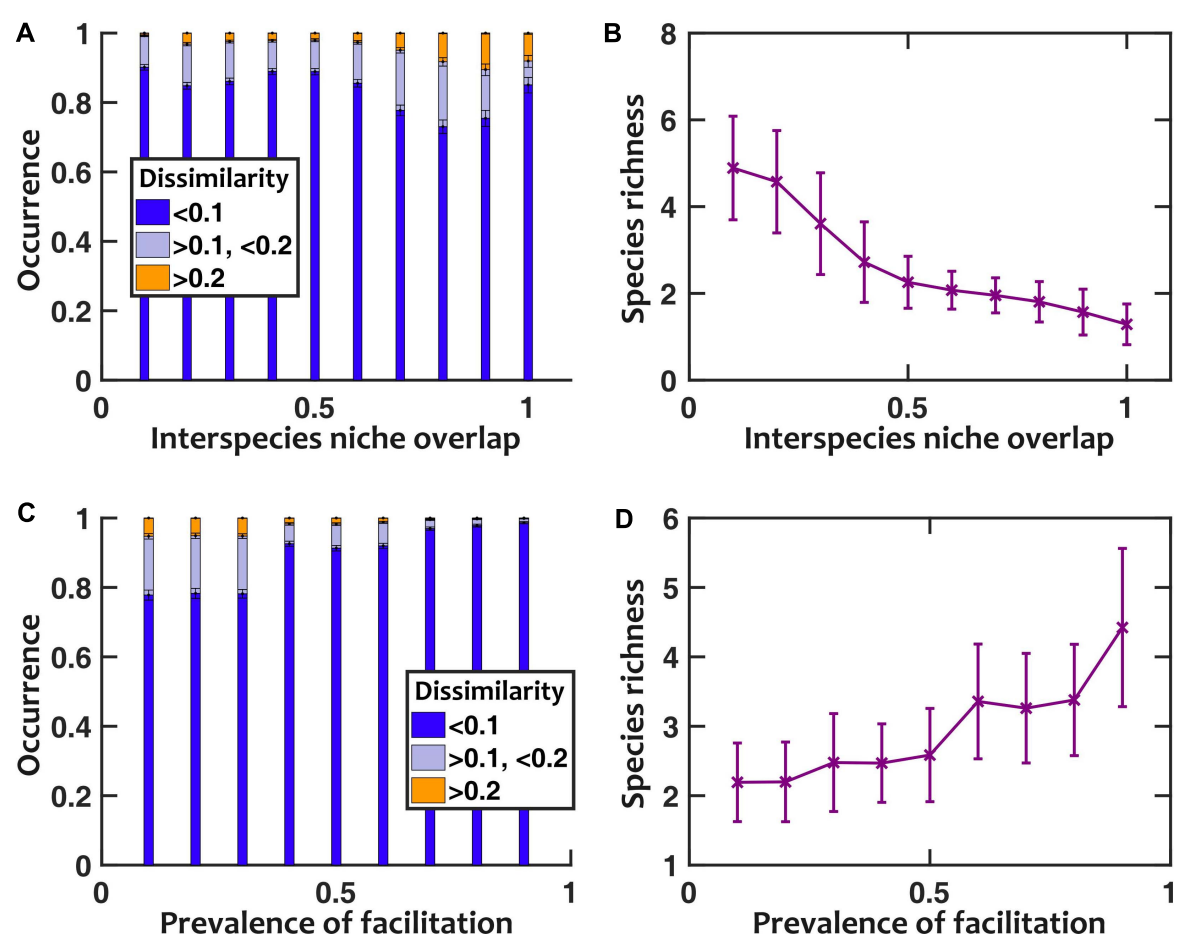

FIGURE 6 | Lower niche overlap and more prevalent facilitation decrease the sensitivity to pH fluctuations. (A) As we artificially increased the strength of niche overlap (by setting all off-diagonal coefficients in the interaction matrix to be a fixed negative number and making that number more negative), the sensitivity to pH fluctuations increased. This trend is disrupted when niche overlap approaches 1, because a majority of communities under such conditions lose interspecies diversity (B). As we increased the prevalence of interspecies facilitation by randomly setting a given fraction of interaction coefficients to be positive, the communities became less sensitive to $\mathrm{pH}$ fluctuations $\mathbf{( C )}$ and more diverse in species richness (D). Here $\mathrm{pH}$ fluctuations are sinusoidal, with $f_{\mathrm{pH}}=0.2 / \mathrm{h}$ and $\Delta \mathrm{pH}=0.5$.

\section{DISCUSSION}

Using empirically measured species properties, we assembled stable in silico communities that show coexistence of nasal bacteria. When these communities were exposed to a fluctuating $\mathrm{pH}$ environment, we observed that the composition of stable communities was only modestly affected. Larger $\mathrm{pH}$ fluctuations increased the deviation, as expected; however, even at a $\mathrm{pH}$ fluctuation of 1 which exceeds the observed temporal variation in the nasal passage, the composition of the majority of communities remained minimally affected. We also found that intermediate frequencies of temporal $\mathrm{pH}$ fluctuations caused the largest deviations in community compositions. Finally, in our results, communities with more facilitation interactions were more robust against $\mathrm{pH}$ fluctuations.

In choosing an appropriate model, one must carefully consider the processes of interest and the required level of abstraction to capture those processes (Momeni et al., 2011; Silverman et al., 2018b). Models originally designed for single species populations have been adapted to characterize microbial communities. Community ecology modeling frameworks designed to understand interactions at the macro scale-in both space and time-have also been applied to microbial populations to study the dynamics of succession and restoration, along with the impact of environmental disturbances (Byrd and Segre, 2016; Gilbert and Lynch, 2019). For example, flux balance analyses, a mainstay in microbial metabolic models, can be modified to describe species interactions within a complex microbial community over time (Larsen et al., 2012; Gerber, 2014; Bucci et al., 2016; Fukuyama et al., 2017; Äijö et al., 2018; Silverman et al., 2018a; Shenhav et al., 2019). To create a predictive model for nasal microbiota, we have extended the generalized Lotka-Volterra (gLV) equations to study the impact of $\mathrm{pH}$ fluctuations on community composition. Generalized LotkaVolterra equations have been previously used to investigate species interactions in the human gut (Fisher and Mehta, 2014; Song et al., 2014) and in a cheese-associated microbial community (Äijö et al., 2018; Song et al., 2014). It has also been similarly extended to describe the impact of environmental fluctuations (antibiotics) on gut microbiota (Stein et al., 2013; Song et al., 2014). For our data, the Lotka-Volterra-type model has proven to-at least to the first-order-capture species growth, interactions, and $\mathrm{pH}$-dependence.

One important aspect of temporal fluctuations is their time scale. Even though in nature the fluctuations are not completely regular, our investigation with sinusoidal temporal fluctuations reveal the time scale at which the influence on community composition is the strongest. Our analysis reveals that the fluctuations are more impactful at intermediate frequencies between two extremes (see section "Materials and Methods"). At very low frequencies of $\mathrm{pH}$ fluctuations, the community dynamics are faster than $\mathrm{pH}$ changes; thus we can assume 
the quasi-static approximation applies. In this regime, the community reaches its stable state locally (in time), and the composition follows the value of $\mathrm{pH}$ at any given time, regardless of the frequency of the $\mathrm{pH}$ fluctuations. In the other extreme, at very high frequencies of $\mathrm{pH}$ fluctuations, the population dynamics cannot follow rapid changes in $\mathrm{pH}$ and essentially the species "see" the average $\mathrm{pH}$. An analysis based on the Wentzel-Kramers-Brillouin (WKB) approximation suggests that in this regime, the magnitude of change in composition (compared to the composition at the average $\mathrm{pH}$ ) is inversely proportional to the $\mathrm{pH}$ fluctuation frequency. Between these two extremes is the zone that exhibits the most change in community composition with $\mathrm{pH}$ fluctuations (Figure $\mathbf{4 B}$ and Supplementary Figure S9B). However, for parameters relevant to the nasal strains we are analyzing, even in this zone the changes in community composition are not drastic.

Our focus in this manuscript is on how composition of stable communities changes when environmental $\mathrm{pH}$ fluctuates. Another relevant question is how fluctuations in $\mathrm{pH}$ affect the process of community assembly. For this, we repeated the community assembly simulations (Supplementary Figures S6,S7), but under an environment in which the $\mathrm{pH}$ temporally fluctuated. Contrary to our expectation, the richness of resulting communities did not monotonically increase with an increase in the amplitude of $\mathrm{pH}$ fluctuations, regardless of fluctuation frequency (Supplementary Figure S10). Instead, we found that richness only changed in a small fraction of in silico nasal communities. Furthermore, in cases with increased richness, S. non-aureus 1 (most facilitative species in our panel) was most frequently added to the community, whereas in cases with decreased richness, S. epidermidis (most inhibitory species in our panel) was most frequently dropped from the community (Supplementary Figure S11). This observation underscores the relative importance of interaction (compared to niche partitioning) in richness outcomes in our model of nasal communities. Our finding is also consistent with predictions about augmentation and colonization resistance using a mediator-explicit model of interactions (Kurkjian et al., 2020).

There are some limitations and simplifications in our study. First, in our investigation we have assumed that fluctuations in $\mathrm{pH}$ are imposed externally (e.g., by the host or the environment). It is also possible that species within the nasal community contribute to the environmental $\mathrm{pH}$. Although outside the scope of this work, we speculate that if species within the community drive the $\mathrm{pH}$ to specific values (Ratzke and Gore, 2018; Ratzke et al., 2018), the impact of external temporal fluctuations of $\mathrm{pH}$ on community composition will be even more diminished. Second, in our model, we assumed that interactions among species remained unchanged at different environmental $\mathrm{pH}$ values. We examined in silico how $\mathrm{pH}$ dependent interaction coefficients might affect our results. For this, we assumed that interaction coefficients changed linearly with $\mathrm{pH}$ in each case (see section "Materials and Methods") and asked how strong the dependency had to be to considerably change the community composition under a fluctuating $\mathrm{pH}$. We observed a significant impact only when the interaction coefficients were strongly $\mathrm{pH}$ dependent (i.e., to the level that the sign of interactions would change within the range of $\mathrm{pH}$ fluctuations) (Supplementary Figure S12).

Our work suggests that a shift in $\mathrm{pH}$ can change the community composition and coexistence (Supplementary Figure S6). This is consistent with previous observations from profiling different locations along the nasal passage (Yan et al., 2013). However, our prediction is that temporal $\mathrm{pH}$ fluctuations often do not cause a major shift in community structure. As a future step, we plan to verify this prediction experimentally by testing how $\mathrm{pH}$ fluctuations affect in vitro nasal communities. If confirmed, our prediction is that the spatial position of sampling and the heterogeneity of the environment will have a stronger effect on community composition compared to the temporal resolution of sampling. The practical implication is that microbiome profiling of nasal microbes may not require a high temporal resolution. We proposed that high throughput sampling of the nasal microbiome along with the corresponding $\mathrm{pH}$ would be an insightful future step to test our predictions.

Finally, one of the main messages of our work is that nasal microbiota is insensitive to temporal fluctuations in $\mathrm{pH}$. It is tantalizing to speculate, when examining other microbial communities, under what conditions this statement is valid. Recent work by Shibasaki et al. (2020) shows that under a fluctuating environment species properties play an important role in community diversity. Our results corroborate their finding. Insensitivity of the members to the environmental fluctuations - as trivial as it may sound-is a defining factor for how sensitive the community is. In the nasal microbiota, species that we are examining are adapted to the nasal environment and the range of $\mathrm{pH}$ fluctuations experienced in this environment is not large. As a result, the community is not majorly affected by $\mathrm{pH}$ fluctuations. On top of this, we also observe that interactions-in particular, facilitation and competitioncan act as stabilizing or de-stabilizing factors for how the community responds to external variations. In other words, facilitation between community members acts as a composition stabilizing factor between populations, which lowers the impact of external fluctuations (Figure 6A). In contrast, inhibition between community members typically exaggerates the changes introduced by external fluctuations (Figure 6C).

\section{DATA AVAILABILITY STATEMENT}

Codes related to this manuscript can be found at: https://github. com/bmomeni/temporal-fluctuations. The raw data supporting the conclusions of this article will be made available by the authors, without undue reservation.

\section{AUTHOR CONTRIBUTIONS}

$\mathrm{SD}, \mathrm{NZ}, \mathrm{Y}-\mathrm{YL}$, and $\mathrm{BM}$ conceived the research. SD and $\mathrm{BM}$ designed the simulations and experiments and wrote the manuscript. SD ran the experiments. SD, MA, SKD, NZ, and BM ran the simulations. SD, MA, SKD, NZ, Y-YL, and BM edited the manuscript. All authors contributed to the article and approved the submitted version. 


\section{FUNDING}

SD was supported by the NIH T32 training grant. Work in the Momeni Lab was supported by a startup fund from Boston College and by an Award for Excellence in Biomedical Research from the Smith Family Foundation. NZ was supported by the China Scholarship Council. Y-YL acknowledges grants R01AI141529, R01HD093761, UH3OD023268, U19AI095219, and U01HL089856 from National Institutes of Health.

\section{REFERENCES}

Abrams, P. A., and Holt, R. D. (2002). The impact of consumer-resource cycles on the coexistence of competing consumers. Theor. Popul. Biol. 62, 281-295. doi: $10.1006 /$ tpbi.2002.1614

Abreu, C. I., Andersen Woltz, V. L., Friedman, J., and Gore, J. (2020). Microbial communities display alternative stable states in a fluctuating environment. PLoS Comput. Biol. 16:e1007934. doi: 10.1371/journal.pcbi.1007934

Äijö, T., Müller, C. L., and Bonneau, R. (2018). Temporal probabilistic modeling of bacterial compositions derived from 16S rRNA sequencing. Bioinformatics 34, 372-380. doi: 10.1093/bioinformatics/btx549

Amarasekare, P. (2003). Competitive coexistence in spatially structured environments: a synthesis. Ecol. Lett. 6, 1109-1122. doi: 10.1046/j.14610248.2003.00530.x

Bomar, L., Brugger, S. D., Yost, B. H., Davies, S. S., and Lemon, K. P. (2016). Corynebacterium accolens releases antipneumococcal free fatty acids from human nostril and skin surface Triacylglycerols. mBio 7:e01725-15. doi: 10 . 1128/mBio.01725-1715

Brugger, S. D., Bomar, L., and Lemon, K. P. (2016). Commensal-pathogen interactions along the human nasal passages. PLoS Pathog. 12:e1005633. doi: 10.1371/journal.ppat.1005633

Brugger, S. D., Eslami, S. M., Pettigrew, M. M., Escapa, I. F., Henke, M. T., Kong, Y., et al. (2020). Dolosigranulum pigrum cooperation and competition in human nasal microbiota. $m$ Sphere 5:e0852-20. doi: 10.1128/mSphere.00852-820

Bucci, V., Tzen, B., Li, N., Simmons, M., Tanoue, T., Bogart, E., et al. (2016). MDSINE: Microbial Dynamical Systems INference Engine for microbiome time-series analyses. Genome Biol. 17:121. doi: 10.1186/s13059-016-0980-6

Byrd, A. L., and Segre, J. A. (2016). Adapting Koch's postulates. Science 351, 224-226. doi: 10.1126/science.aad6753

Cespedes, C., Saïd-Salim, B., Miller, M., Lo, S. H., Kreiswirth, B. N., Gordon, R. J., et al. (2005). The clonality of Staphylococcus aureus nasal carriage. J. Infect. Dis. 191, 444-452. doi: 10.1086/427240

Chesson, P. (2000). Mechanisms of maintenance of species diversity. Annu. Rev. Ecol. Syst. 31, 343-366. doi: 10.1146/annurev.ecolsys.31.1.343

De Vos, M. G. J., Zagorski, M., McNally, A., and Bollenbach, T. (2017). Interaction networks, ecological stability, and collective antibiotic tolerance in polymicrobial infections. Proc. Natl. Acad. Sci. U.S.A. 114, 10666-10671. doi: 10.1073/pnas.1713372114

Dedrick, S., Akbari, M. J., Dyckman, S., Zhao, N., Liu, Y.-Y., and Momeni, B. (2020). Impact of temporal $\mathrm{pH}$ fluctuations on the coexistence of nasal bacteria. bioRxiv [Preprint], doi: 10.1101/2020.09.15.298778

Eddison, J. C., and Ollason, J. G. (1978). Diversity in constant and fluctuating environments [11]. Nature 275, 309-310. doi: 10.1038/275309a0

Escapa, I. F., Chen, T., Huang, Y., Gajare, P., Dewhirst, F. E., and Lemon, K. P. (2018). New insights into human nostril microbiome from the expanded human oral microbiome database (eHOMD): a resource for the microbiome of the human aerodigestive tract. mSystems 3:e0187-18. doi: 10.1128/mSystems. 00187- 118

Fisher, C. K., and Mehta, P. (2014). Identifying keystone species in the human gut microbiome from metagenomic timeseries using sparse linear regression. PLoS One 9:e0102451. doi: 10.1371/journal.pone.0102451

Fukuyama, J., Rumker, L., Sankaran, K., Jeganathan, P., Dethlefsen, L., Relman, D. A., et al. (2017). Multidomain analyses of a longitudinal human microbiome

\section{ACKNOWLEDGMENTS}

We would like to thank Dr. Katherine Lemon for kindly sharing the nasal bacterial strains with us. This manuscript has been released as a pre-print at (Dedrick et al., 2020).

\section{SUPPLEMENTARY MATERIAL}

The Supplementary Material for this article can be found online at: https://www.frontiersin.org/articles/10.3389/fmicb. 2021.613109/full\#supplementary-material

intestinal cleanout perturbation experiment. PLoS Comput. Biol. 13:e1005706. doi: 10.1371/journal.pcbi.1005706

Gerber, G. K. (2014). The dynamic microbiome. FEBS Lett. 588, 4131-4139. doi: 10.1016/j.febslet.2014.02.037

Gilbert, J. A., and Lynch, S. V. (2019). Community ecology as a framework for human microbiome research. Nat. Med. 25, 884-889. doi: 10.1038/s41591-0190464-9

Goldford, J. E., Lu, N., Bajiæ, D., Estrela, S., Tikhonov, M., Sanchez-Gorostiaga, A., et al. (2018). Emergent simplicity in microbial community assembly. Science 361, 469-474. doi: 10.1126/science.aat1168

Grover, J. P. (1988). Dynamics of competition in a variable environment: experiments with two diatom species. Ecology 69, 408-417. doi: 10.2307/ 1940439

Hehar, S. S., Mason, J. D. T., Stephen, A. B., Washington, N., Jones, N. S., Jackson, S. J., et al. (2001). Twenty-four hour ambulatory nasal pH monitoring. Clin. Otolaryngol. Allied Sci. 24, 24-25. doi: 10.1046/j.1365-2273. 1999.00190.x

Iwase, T., Uehara, Y., Shinji, H., Tajima, A., Seo, H., Takada, K., et al. (2010). Staphylococcus epidermidis Esp inhibits Staphylococcus aureus biofilm formation and nasal colonization. Nature 465, 346-349. doi: 10.1038/ nature09074

Jiang, L., and Morin, P. J. (2007). Temperature fluctuation facilitates coexistence of competing species in experimental microbial communities. J. Anim. Ecol. 76, 660-668. doi: 10.1111/j.1365-2656.2007.01252.x

Johannessen, M., Sollid, J. E., and Hanssen, A.-M. (2012). Host- and microbe determinants that may influence the success of $S$. aureus colonization. Front. Cell. Infect. Microbiol. 2:56. doi: 10.3389/fcimb.2012.00056

Kaspar, U., Kriegeskorte, A., Schubert, T., Peters, G., Rudack, C., Pieper, D. H., et al. (2016). The culturome of the human nose habitats reveals individual bacterial fingerprint patterns. Environ. Microbiol. 18, 2130-2142. doi: 10.1111/ 1462-2920.12891

Kiryukhina, N. V., Melnikov, V. G., Suvorov, A. V., Morozova, Y. A., and Ilyin, V. K. (2013). Use of Corynebacterium pseudodiphtheriticum for elimination of Staphylococcus aureus from the nasal cavity in volunteers exposed to abnormal microclimate and altered gaseous environment. Probiot. Antimicrob. Proteins 5, 233-238. doi: 10.1007/s12602-013-9147-x

Kremer, C. T., and Klausmeier, C. A. (2013). Coexistence in a variable environment: eco-evolutionary perspectives. J. Theor. Biol. 339, 14-25. doi: 10.1016/j.jtbi. 2013.05.005

Krismer, B., Liebeke, M., Janek, D., Nega, M., Rautenberg, M., Hornig, G., et al. (2014). Nutrient limitation governs Staphylococcus aureus metabolism and niche adaptation in the human nose. PLoS Pathog. 10:e1003862. doi: 10.1371/ journal.ppat.1003862

Krismer, B., Weidenmaier, C., Zipperer, A., and Peschel, A. (2017). The commensal lifestyle of Staphylococcus aureus and its interactions with the nasal microbiota. Nat. Rev. Microbiol. 15, 675-687. doi: 10.1038/nrmicro.20 17.104

Kurkjian, H. M., Akbari, M. J., and Momeni, B. (2020). The impact of interactions on invasion and colonization resistance in microbial communities. bioRxiv [Preprint], doi: 10.1101/2020.06.11.146571

Larsen, P. E., Field, D., and Gilbert, J. A. (2012). Predicting bacterial community assemblages using an artificial neural network approach. Nat. Methods 9, 621-625. doi: 10.1038/nmeth.1975 
Man, W. H., de Steenhuijsen Piters, W. A. A., and Bogaert, D. (2017). The microbiota of the respiratory tract: gatekeeper to respiratory health. Nat. Rev. Microbiol. 15, 259-270. doi: 10.1038/nrmicro.2017.14

Margolis, E., Yates, A., and Levin, B. R. (2010). The ecology of nasal colonization of Streptococcus pneumoniae, Haemophilus influenzae and Staphylococcus aureus: the role of competition and interactions with host's immune response. BMC Microbiol. 10:59. doi: 10.1186/1471-2180-10-59

Momeni, B., Chen, C.-C., Hillesland, K. L., Waite, A., and Shou, W. (2011). Using artificial systems to explore the ecology and evolution of symbioses. Cell. Mol. Life Sci. 68, 1353-1368. doi: 10.1007/s00018-011-0649-y

Momeni, B., Xie, L., and Shou, W. (2017). Lotka-Volterra pairwise modeling fails to capture diverse pairwise microbial interactions. eLife 6:e25051. doi: 10.7554/ eLife. 25051

Mounier, J., Monnet, C., Vallaeys, T., Arditi, R., Sarthou, A.-S., Hélias, A., et al. (2008). Microbial interactions within a cheese microbial community. Appl. Environ. Microbiol. 74, 172-181. doi: 10.1128/AEM.01338-07

Niehaus, L., Boland, I., Liu, M., Chen, K., Fu, D., Henckel, C., et al. (2019). Microbial coexistence through chemical-mediated interactions. Nat. Commun. 10:2052. doi: 10.1038/s41467-019-10062-x

Proctor, D. M., and Relman, D. A. (2017). The landscape ecology and microbiota of the human nose, mouth, and throat. Cell Host Microb. 21, 421-432. doi: 10.1016/J.CHOM.2017.03.011

Ratzke, C., Denk, J., and Gore, J. (2018). Ecological suicide in microbes. Nat. Ecol. Evol. 2, 867-872. doi: 10.1038/s41559-018-0535-531

Ratzke, C., and Gore, J. (2018). Modifying and reacting to the environmental pH can drive bacterial interactions. PLoS Biol. 16:e2004248. doi: 10.1371/journal. pbio. 2004248

Regev-Yochay, G., Raz, M., Dagan, R., Porat, N., Shainberg, B., Pinco, E., et al. (2004). Nasopharyngeal carriage of Streptococcus pneumoniae by adults and children in community and family settings. Clin. Infect. Dis. 38, 632-639. doi: $10.1086 / 381547$

Relman, D. A. (2012). The human microbiome: ecosystem resilience and health. Nutr. Rev. 70, S2-S9. doi: 10.1111/j.1753-4887.2012.00489.x

Shenhav, L., Furman, O., Briscoe, L., Thompson, M., Silverman, J. D., Mizrahi, I., et al. (2019). Modeling the temporal dynamics of the gut microbial community in adults and infants. PLOS Comput. Biol. 15:e1006960. doi: 10.1371/journal. pcbi. 1006960

Shibasaki, S., Mobilia, M., and Mitri, S. (2020). Microbial species interactions determine community diversity in fluctuating environments. bioRxiv [Preprint], doi: 10.1101/2020.07.22.216010

Silverman, J. D., Durand, H. K., Bloom, R. J., Mukherjee, S., and David, L. A. (2018a). Dynamic linear models guide design and analysis of microbiota studies within artificial human guts. Microbiome 6:202. doi: 10.1186/s40168018-0584-3
Silverman, J., Shenhav, L., Halperin, E., Mukherjee, S., and David, L. A. (2018b). Statistical considerations in the design and analysis of longitudinal microbiome studies. bioRxiv doi: 10.11164/jjsps.27.3_633_1

Song, H.-S., Cannon, W., Beliaev, A., and Konopka, A. (2014). Mathematical modeling of microbial community dynamics: a methodological review. Processes 2, 711-752. doi: 10.3390/pr2040711

Stein, R. R., Bucci, V., Toussaint, N. C., Buffie, C. G., Rätsch, G., Pamer, E. G., et al. (2013). Ecological modeling from time-series inference: insight into dynamics and stability of intestinal microbiota. PLoS Comput. Biol. 9:e1003388. doi: 10. 1371/journal.pcbi.1003388

Uehara, Y., Nakama, H., Agematsu, K., Uchida, M., Kawakami, Y., Abdul Fattah, A., et al. (2000). Bacterial interference among nasal inhabitants: eradication of Staphylococcus aureus from nasal cavities by artificial implantation of Corynebacterium sp. J. Hosp. Infect. 44, 127-133. doi: 10.1053/ JHIN.1999.0680

Venturelli, O. S., Carr, A. V., Fisher, G., Hsu, R. H., Lau, R., Bowen, B. P., et al. (2018). Deciphering microbial interactions in synthetic human gut microbiome communities. Mol. Syst. Biol. 14:e8157. doi: 10.15252/MSB.2017 8157

Washington, N., Steele, R. J., Jackson, S., Bush, D., Mason, J., Gill, D., et al. (2000). Determination of baseline human nasal $\mathrm{pH}$ and the effect of intranasally administered buffers. Int. J. Pharm. 198, 139-146. doi: 10.1016/S0378-5173(99) 00442-441

Wertheim, H. F., Vos, M. C., Ott, A., van Belkum, A., Voss, A., Kluytmans, J. A., et al. (2004). Risk and outcome of nosocomial Staphylococcus aureus bacteraemia in nasal carriers versus non-carriers. Lancet 364, 703-705. doi: 10.1016/S0140-6736(04)16897-16899

Yan, M., Pamp, S. J., Fukuyama, J., Hwang, P. H., Cho, D.-Y., Holmes, S., et al. (2013). Nasal microenvironments and interspecific interactions influence nasal microbiota complexity and $S$. aureus carriage. Cell Host Microb. 14, 631-640. doi: 10.1016/J.CHOM.2013.1 1.005

Conflict of Interest: The authors declare that the research was conducted in the absence of any commercial or financial relationships that could be construed as a potential conflict of interest.

Copyright (C) 2021 Dedrick, Akbari, Dyckman, Zhao, Liu and Momeni. This is an open-access article distributed under the terms of the Creative Commons Attribution License (CC BY). The use, distribution or reproduction in other forums is permitted, provided the original author(s) and the copyright owner(s) are credited and that the original publication in this journal is cited, in accordance with accepted academic practice. No use, distribution or reproduction is permitted which does not comply with these terms. 